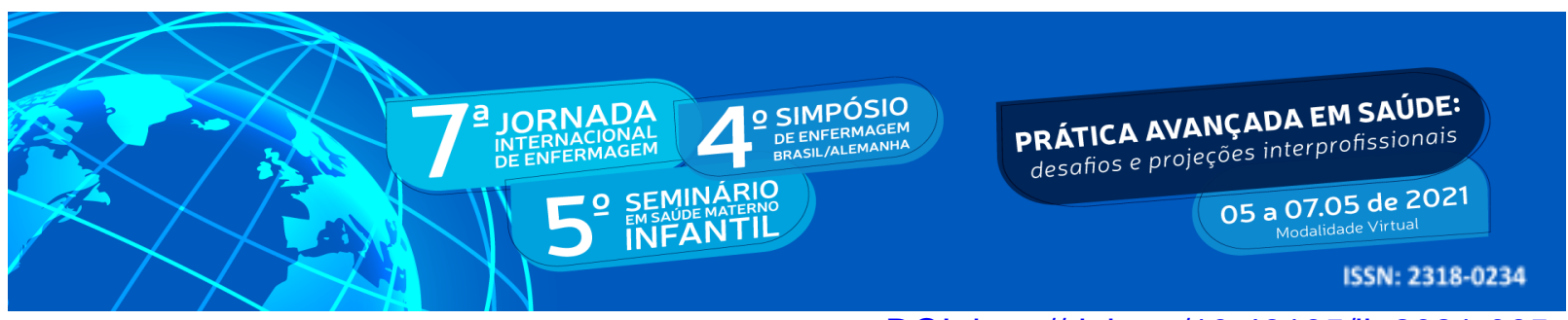

DOI: http://doi.org/10.48195/jie2021-065

\title{
USO DO PRONTUÁRIO ELETRÔNICO DO CIDADÃO NA CONSULTA DE ENFERMAGEM EM PUERICULTURA: RELATO DE EXPERIÊNCIA
}

\author{
Ana Karolina de Lima Alves da Silva ${ }^{1}$; Isabel Pires Barra ${ }^{2}$; Magnólia Carvalho Aquino \\ Gonzaga $^{3}$; Renis Cineide Pereira de Moura ${ }^{4}$; Paula Fernanda Brandão Batista dos \\ Santos $^{5}$
}

\section{RESUMO}

Objetivo: Relatar a experiência vivenciada durante o estágio supervisionado em uma Unidade de Saúde da Família sobre a utilização do Prontuário Eletrônico do Cidadão como uma ferramenta para a consulta de enfermagem em puericultura. Métodos: Trata-se de um relato de experiência obtido através de consultas realizadas durante o período de Estágio Supervisionado, realizado em Natal no Rio Grande do Norte, no período de janeiro a fevereiro de 2021. Para a coleta dos dados foram utilizadas as seguintes técnicas: prontuário eletrônico do cidadão, participação nas atividades clínicas, consulta à caderneta da criança. Resultados: Foram realizadas 64 consultas durante os meses de janeiro e fevereiro, observou-se que o sistema possibilita o acesso rápido às informações e intervenções realizadas, traz melhoria na efetividade do cuidado e na qualidade das informações. Conclusão: Conclui-se que o prontuário eletrônico possibilita o aprimoramento da comunicação entre os profissionais da atenção básica, permitindo a avaliação e o acompanhamento do crescimento e desenvolvimento.

Palavras-chave: Prontuário eletrônico do cidadão; Puericultura; Enfermagem.

\begin{abstract}
Objective: To report the experience lived during the supervised internship at a Family Health Unit on the use of the Electronic Citizen's Record as a tool for nursing consultation in childcare. Methods: This is an experience report obtained through consultations carried out during the period of Supervised Internship, held in Natal, Rio Grande do Norte, from January to February 2021. For data collection, the following techniques were used : citizen's electronic medical record, participation in clinical activities, consultation of the child's handbook. Results: 64 consultations were carried out during the months of January and February, it was observed that the system allows quick access to the information and interventions performed, it improves the effectiveness of care and the quality of information. Conclusion: It is concluded that the electronic medical record enables the improvement of communication between primary care professionals, allowing the assessment and monitoring of growth and development.
\end{abstract}

\footnotetext{
${ }^{1}$ Graduanda do Curso de Enfermagem pela Universidade Federal do Rio Grande do Norte - UFRN. E-mail: anaklasilva@gmail.com;

${ }^{2}$ Graduanda do Curso de Enfermagem pela Universidade Federal do Rio Grande do Norte - UFRN. E-mail: barraisa20@gmail.com;

${ }^{3}$ Enfermeira da Estratégia de Saúde da Família, Natal, RN. E-mail: magnoliagonzaga@hotmail.com;

${ }^{4}$ Enfermeira da Estratégia de Saúde da Família, Natal, RN. E-mail: renismoura@hotmail.com;

5 Professora do Departamento de Enfermagem da Universidade Federal do Rio Grande do Norte. E-mail: paulafernandabbs@gmail.com;
} 


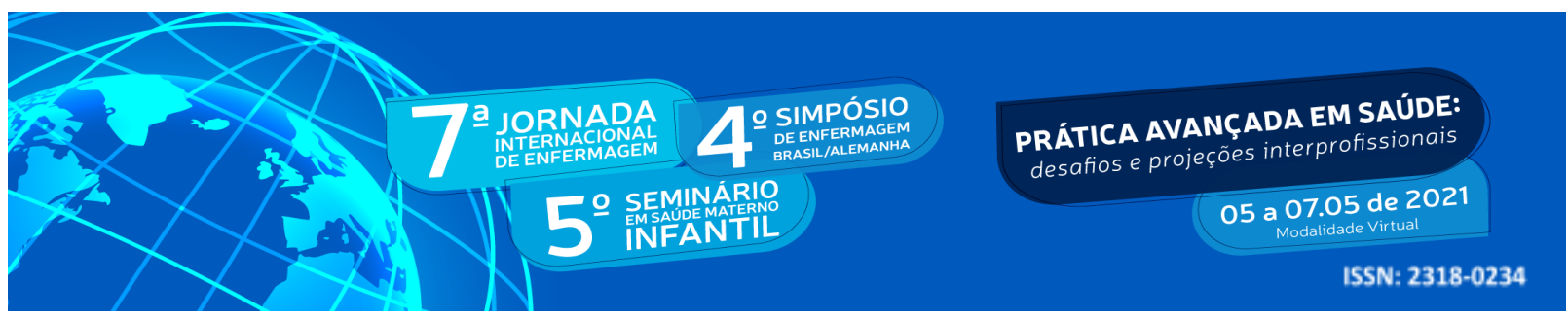

Key Words: Citizen's electronic medical record; Childcare; Nursing.

\section{INTRODUÇÃO}

Os avanços tecnológicos na área da saúde são imprescindíveis no compartilhamento de informações que melhorem a assistência à saúde do paciente, difundindo dados com qualidade e reduzindo possíveis erros (MOURÃO E NEVES, 2007).

"A palavra prontuário deriva do latim "promptuariu" que significa lugar onde se guarda aquilo que deve estar à mão, o que pode ser necessário a qualquer momento" (COFEN, 2016).

Trata-se de um documento único constituído de um conjunto de informações, de sinais e de imagens registradas, geradas a partir de fatos, acontecimentos e situações sobre a saúde do paciente e a assistência a ele prestada, de caráter legal, sigiloso e científico, que possibilita a comunicação entre membros de uma equipe multiprofissional (CONSELHO FEDERAL DE MEDICINA, 2002).

É no prontuário do paciente que estão contidas as informações que refletem a assistência prestada durante sua consulta. É nele que devem ser registrados as preocupações, reações e condições biopsicossociais dos indivíduos e também as condutas profissionais implementadas..

Até poucos anos atrás o prontuário em papel era a realidade de grande parte das unidades básicas de saúde pelo Brasil. $\mathrm{O}$ uso do prontuário de papel representava diversas dificuldades para a assistência, principalmente no que tange ao tempo despendido para assistir ao indivíduo e registrar seus dados, levando muitos profissionais a resumir as informações do paciente na hora do registro em prontuário. O risco de danos ou extravio dos prontuários era considerável, tendo em vista a quantidade de prontuários armazenados em um só local e o manuseio desse material por diversos profissionais.

A portaria $\mathrm{n}^{\circ} 1.142$, de 10 de Julho de 2013 que implementa o Sistema de Informação em Saúde para a Atenção Básica (SISAB) traz o que conhecemos por e-SUS AB, que objetiva 


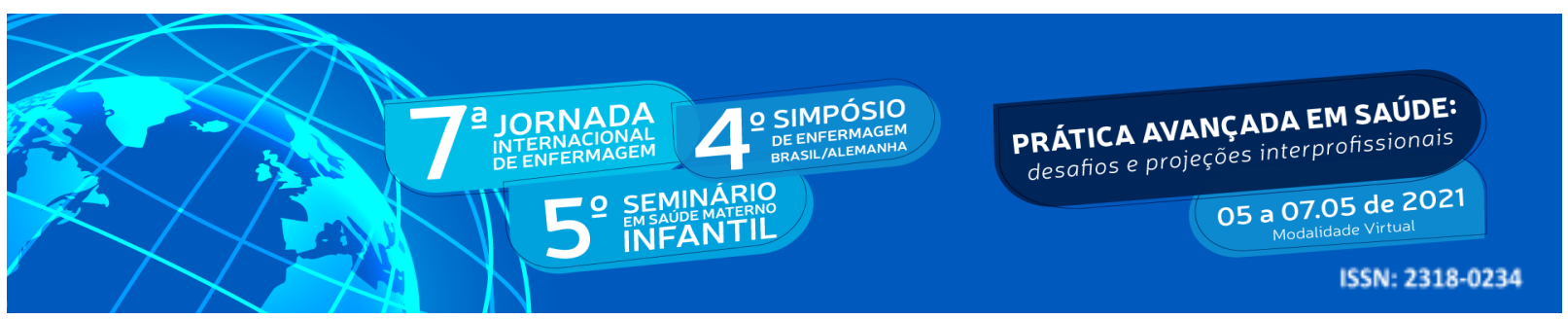

a informatização das unidades de saúde. (MINISTÉRIO DA SAÚDE, 2013). O lançamento do Prontuário Eletrônico do Cidadão (PEC), ainda em versão mais simplificada, caracterizou-se como o início da informatização da atenção básica. (LIMA et al., 2018). Na Atenção Básica caracterizou-se como enorme avanço na assistência à saúde do usuário, capaz de aumentar, significativamente, a produtividade e os índices de qualidade e satisfação das pessoas usuárias, além de possibilitar manter um registro de fácil acesso.

O PEC é um sistema que tem a função de unir todos os dados do paciente, feitos por diferentes profissionais da área da saúde, além de ser uma ferramenta para apoiar os usuários, fornecendo acesso aos dados completos e corretos. Sendo o seu principal objetivo, permitir a qualidade no atendimento ao paciente e sua assistência, em lugares e cenários distintos (OLIVEIRA, 2013; LAHM; CARVALHO, 2015.).

Segundo Pinto (2006) o prontuário eletrônico apresenta vantagens, como: a redução de tempo de atendimento, impedimento de que um setor/profissional mantenha consigo o prontuário impossibilitando o prosseguimento do processo de atendimento, possibilidade de reconstrução histórica, melhoria da legibilidade, facilidade na organização e no acesso às informações, diminuindo grandes espaços para guarda e arquivo dos documentos, promoção da comunicação entre o paciente e a equipe de saúde.

Assim como o PEC apresenta diversos benefícios para assistência ao paciente e para os profissionais, é importante ressaltar que as desvantagens devem ser levadas em consideração e principalmente buscar amenizá-las o máximo possível.

Apesar da implantação do PEC, mantém-se a necessidade de armazenamento adequado dos prontuários em papel, a fim de evitar extravio e avarias. O prontuário em papel permanece sendo um documento usado para fins legais quando não há definição jurídica para documentação de meio eletrônico. Assim como, perdura a necessidade de qualificação e atualizações frequentes dos profissionais, de investimentos e manutenções no sistema de informação. A possibilidade de falta de energia e os obstáculos impostos pelos profissionais para não se adaptarem aos novos instrumentos de trabalho. (BROCHETTO et al., 2015). 


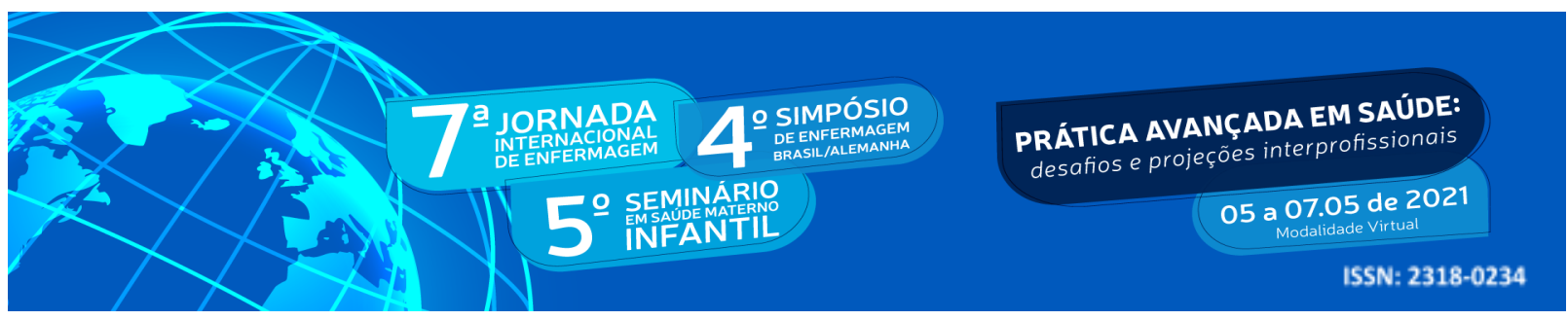

\section{OBJETIVO}

Relatar a experiência da utilização do Prontuário Eletrônico do Cidadão na consulta de acompanhamento do crescimento e desenvolvimento infantil durante o estágio supervisionado em uma Unidade de Saúde da Família.

\section{METODOLOGIA}

O relato de experiência é uma ferramenta de pesquisa descritiva que apresenta uma reflexão sobre algo vivenciado. A escolha por apresentar esse relato de experiência parte da compreensão que se comunga de que "o processo de conhecer o mundo anda de mãos dadas com sua transformação" (BRANDÃO, STRECK, 2006). Nesse sentido, apresentar esse relato de experiência permite compartilhar experiência que demonstrou mudança significativa no processo de trabalho do enfermeiro e que pode servir de aprendizado não apenas para aqueles que o vivenciaram, mas para todos os demais que possam ter acesso ao que aqui se apresenta.

É preciso reconhecer que as experiências são processos individuais, mas também coletivos e que são ao mesmo tempo sócio-histórico, dinâmicos e complexos. Pode-se dizer que as experiências não são simples ações, fatos pontuais, mas vivências imbuídas de dinamismo, complexidade, movidas pela necessidade de promover transformações. (HOLLIDAY, 2006) Na leitura de uma experiência é preciso que se considere o contexto em que ela ocorre e as situações particulares em que ela repousa para que se possa extrair o conhecimento e aprendizado que os autores buscam comunicar.

As experiências se constituem, portanto, em um processo inédito e irrepetível, e por isso, cada uma detém uma riqueza que deve ser aproveitada precisamente por sua particularidade, e por isso faz-se necessário compreender estas experiências, extrair seus ensinamentos e também comunicá-los (HOLLIDAY, 2006)

Para a coleta dos dados foram utilizadas as seguintes técnicas: prontuário eletrônico do cidadão, participação nas atividades clínicas, consulta à caderneta da criança. 


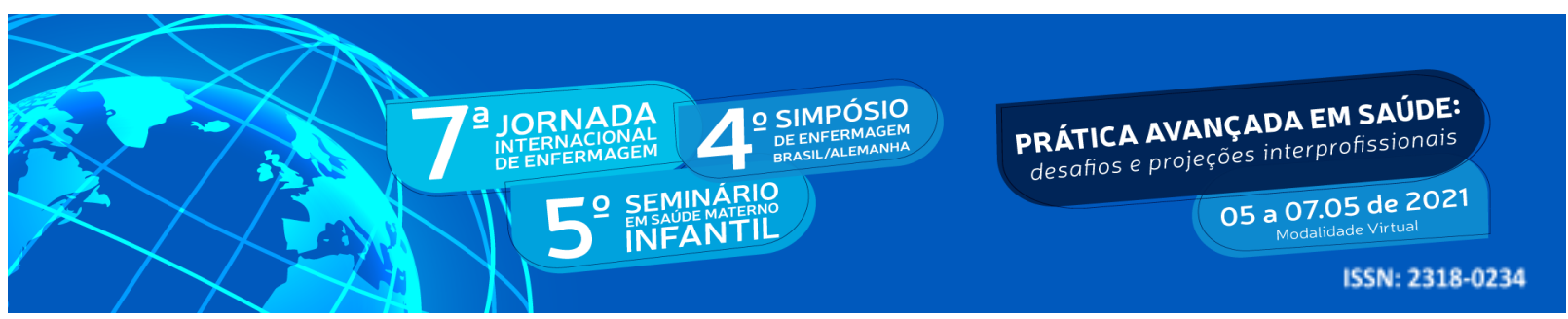

O relato de experiência aqui apresentado foi obtido através de consultas realizadas durante o período de Estágio Supervisionado I - O processo de trabalho do enfermeiro na Atenção Básica de Saúde, do curso de graduação em Enfermagem da Universidade Federal do Rio Grande do Norte, realizado em uma Unidade de Saúde da Família, em Natal no Rio Grande do Norte, em 2021. Nesta experiência, foi vivenciada a utilização do prontuário eletrônico do cidadão como uma ferramenta para as consultas de enfermagem em puericultura.

\section{RESULTADOS E DISCUSSÃO}

As consultas de acompanhamento do crescimento e desenvolvimento da criança de 0 a 10 anos aconteceram nas segundas-feiras e terças-feiras no período de janeiro e fevereiro. Em cada um destes dias são agendadas 6 crianças e são deixadas 6 vagas para demanda espontânea. As consultas são agendadas com antecedência na recepção utilizando o PEC. No dia da consulta o atendimento é realizado com hora marcada.

De acordo com relatório extraído do PEC, no mês de janeiro e fevereiro, foram realizadas 64 consultas de acompanhamento do crescimento e desenvolvimento da criança, pela enfermeira supervisora, juntamente com as alunas em estágio, sendo 48 consultas com crianças menores de 1 ano, 9 com crianças de 1 ano, 4 com crianças de 2 anos, 2 consultas com crianças de 3 anos, 2 consultas com crianças de 4 anos e 2 com crianças de 5 à 9 anos. $\mathrm{O}$ relatório também mostrou que 28 crianças atendidas estavam em aleitamento materno exclusivo, 3 em aleitamento materno predominante, $20 \mathrm{em}$ aleitamento materno complementar e 4 crianças com aleitamento inexistente.

Para a realização das consultas em puericultura, o PEC possui uma ferramenta de acompanhamento do crescimento e desenvolvimento da criança, podendo o profissional de saúde fazer o acompanhamento da criança até aos 10 anos de idade. Ao habilitar o campo de Puericultura, pode-se inserir as informações em blocos, sobre o pré-natal, parto, nascimento, antropometria e sinais vitais, o próprio sistema já calcula o Índice de Massa Corporal - IMC.

No PEC pode-se também registrar os dados referentes às alterações fenotípicas, fatores de riscos sociais e ambientais, e disponibiliza a avaliação do desenvolvimento 


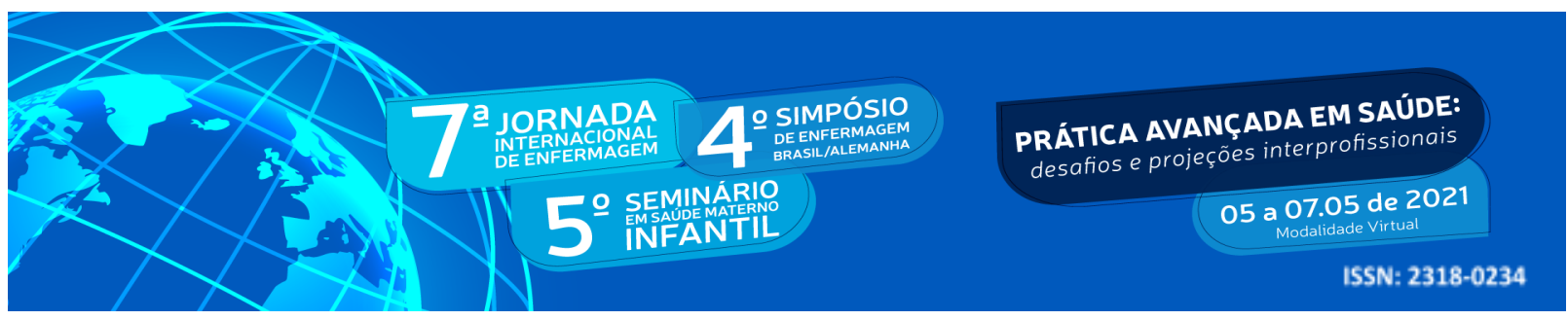

neuropsicomotor, sendo possível avaliar os reflexos primitivos e os marcos do desenvolvimento da criança de acordo com a idade em meses, além disso, auxilia o profissional como avaliar determinado marco, e dá a classificação da avaliação, se a criança se encontra em atraso ou não no seu desenvolvimento.

Ademais, o PEC permite o registro do resultado de exames, como os testes de triagem, porém, percebeu-se que no sistema não está incluído o exame de triagem teste da linguinha, o que fez com que o registrássemos no campo de "Avaliação". Após a realização do exame físico e a avaliação e interpretação dos dados obtidos, as informações também são registradas na caderneta da criança. São realizadas orientações aos pais em relação aos cuidados com a criança, como por exemplo na prevenção de acidentes e introdução alimentar, além da avaliação da situação vacinal da criança.

Sempre orientamos os pais sobre a importância da vacinação para prevenção de doenças. Após as explicações e orientações realizadas, a consulta é encerrada e essa família já sai do consultório com o retorno marcado ou encaminhada para atendimento como outro profissional da equipe, como médico ou dentista.

O PEC se constitui em uma importante ferramenta para as consultas de puericultura, pois além de ser possível registrar todas essas informações para o acompanhamento do desenvolvimento da criança, estes dados comporão as tabelas e gráficos, onde é possível analisar a curva de crescimento da criança, da mesma forma que os gráficos presentes na caderneta da criança.

Considerando que o PEC fornece elementos para registro mais amplo, uma vez que exige o preenchimento e avaliação de alguns dados, além de orientar profissional como fazer a avaliação neuropsicomotor da criança e os marcos do desenvolvimento, o que antes no prontuário de papel não existia, pois eram páginas em branco em que o profissional fazia a sua evolução, então muita informação era perdida.

Como o PEC exige um tempo maior para o seu preenchimento, para facilitar a realização dos registros nas consultas no PEC, observou-se a necessidade de elaborar um material para otimizar o tempo e facilitar o atendimento às questões gerais da saúde da criança. Então, foram criados roteiros especificamente para cada faixa etária: consulta ao recém-nascido, crianças de 1 a 4 meses, 4 a 6 meses, 6 a 9 meses, 9 a 12 meses e de 12 a 24 


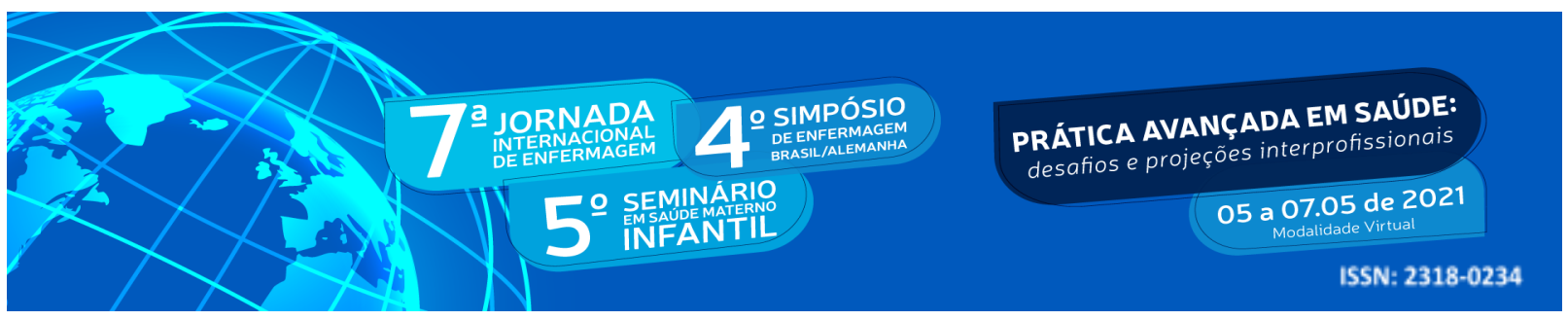

meses. Além disso, foram adicionadas orientações relacionadas aos cuidados com a criança, prevenção de acidentes domésticos e introdução de alimentos de acordo com a faixa etária. Tudo isso para possibilitar uma maior otimização do tempo para o registro destas informações e possibilitar um maior tempo de interação entre o profissional e a família.

As dificuldades encontradas estiveram relacionadas à realização da consulta em si, como também no uso do PEC. No primeiro caso estava relacionado ao exame físico da criança, na etapa de ausculta cardíaca e pulmonar, no que se refere ao PEC, se exige maior tempo durante as consultas e uma boa conexão com a internet para que se efetive o seu uso. Por vezes, já houve momentos de queda da conexão da internet ou queda de energia durante a realização da consulta, sendo necessário anotar os dados em um papel para que, posteriormente, se fizesse o registro da consulta no PEC em atendimento tardio.

Salientamos, que o PEC não prevê o uso de classificações diagnósticas para enfermagem Nele são dispostos a classificação internacional dos problemas de saúde na atenção básica, que deve ser inserido no campo motivo da consulta e problema e/ou condição detecctada. No entanto, enquanto espaço de registro, planejamento e avaliação do cuidado de enfermagem, faz-se necessário buscar inserir elementos que auxiliem esse profissional no seu processo de sistematização da assistência de enfermagem. Assim, contribui-se para o avanço da profissão, da sistematização da assistência de enfermagem no campo da atenção básica fortalecendo a ação profissional do enfermeiro.

Ressaltamos que o PEC possui um grande potencial para gerar relatórios individuais quanto a população de determinada equipe ou de todos os usuários cadastrados na unidade básica, permitindo uma maior vigilância desta população, acompanhamento das condições de saúde, como por exemplo, as vacinas atrasadas.

\section{CONCLUSÃO}

O prontuário eletrônico do cidadão é uma ferramenta eficaz durante as consultas de acompanhamento de crescimento e desenvolvimento, podendo servir como um instrumento de vigilância do cuidado. O enfermeiro, através da consulta de enfermagem à criança, 


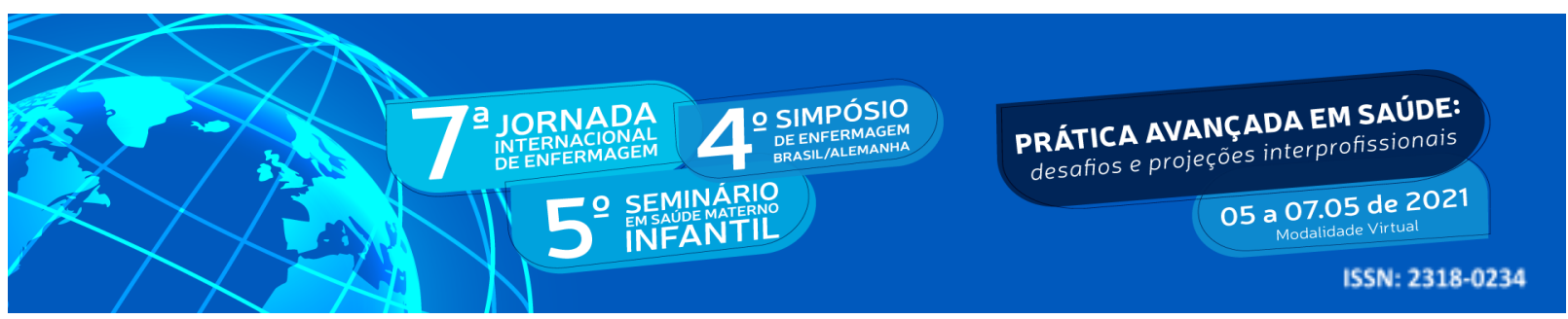

desempenha um papel fundamental no que se refere à promoção da saúde infantil, no acompanhamento do crescimento e desenvolvimento infantil.

O prontuário eletrônico é um sistema que possibilita o acesso rápido às informações e intervenções realizadas, traz melhoria na efetividade do cuidado e na qualidade das informações, aprimoramento na comunicação entre os profissionais da atenção básica, permitindo a avaliação e o acompanhamento do crescimento e desenvolvimento, estado nutricional e vacinação. Alerta o profissional em caso de alteração ou atraso no desenvolvimento, além de acompanhar os gráficos de peso, comprimento, perímetro cefálico e IMC. Com essa ferramenta o processo de trabalho do enfermeiro no cuidado à criança ganha uma outra dimensão, em que a informação torna-se mais facilmente acessível, disponível e capaz de gerar indicadores que vão nortear as ações profissionais.

Ao mesmo tempo, a experiência aqui relatada ressalta que deve-se também criar estratégias que possam favorecer o uso oportuno, rápido e necessário da ferramenta durante a consulta de forma a não implicar numa maior dedicação do tempo do profissional no registro destas informações em detrimento do atendimento da criança e família. Ao mesmo tempo, é importante que o enfermeiro registre no campo de Avaliação, os diagnósticos de enfermagem e no campo Plano as prescrições de enfermagem, conduzindo sua consulta a partir da sistematização da assistência de enfermagem à criança.

\section{REFERÊNCIAS}

BRASIL. Ministério da Saúde. Portaria $\mathbf{N}^{\mathbf{0}}$ 1.412,de 10 de julho de 2013. Disponível em: $<$ http://bvsms.saude.gov.br/bvs/saudelegis/gm/2013/prt1412_10 07 2013.html >.

BROCHeTTO, A. D et al. Prontuário eletrônico do paciente (PEP): análise em hospital da serra gaúcha (RS). Revista Eletrônica Gestão \& Saúde. Vol. 6 (Supl. 3). Abril, 2015

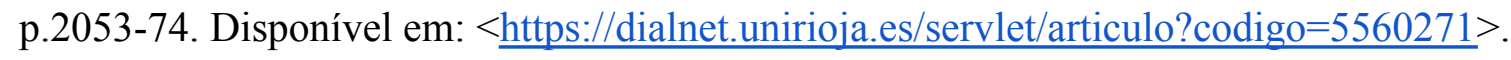

CONSELHO FEDERAL DE ENFERMAGEM. Guia de recomendações para registro de enfermagem no prontuário e outros documentos de enfermagem. Brasília: COFEN, 2016. Disponível

em: $<$ http://www.cofen.gov.br/wp-content/uploads/2016/06/RESOLUCÃO-COFEN-Nº-0514-201 6-GUIA-DE-RECOMENDACÕES-versão-web.pdf $>$. 


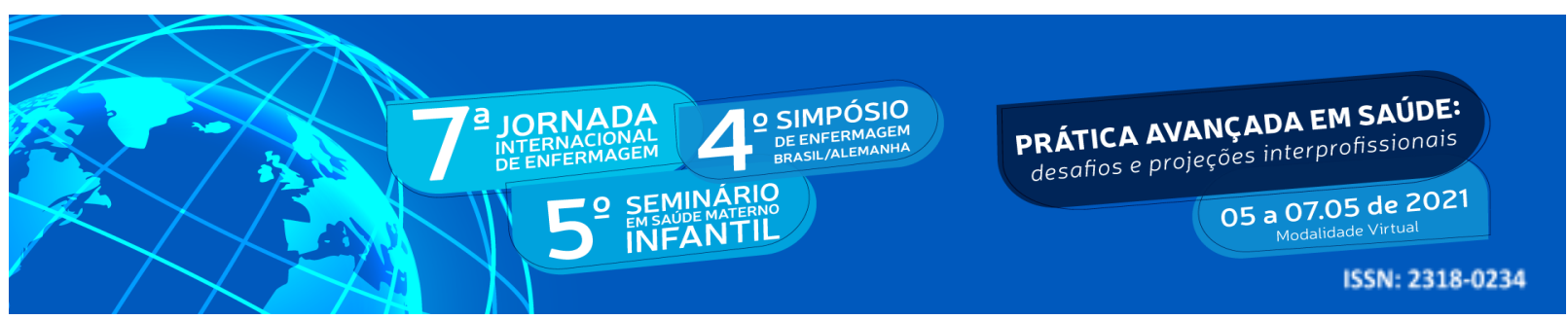

CONSELHO FEDERAL DE MEDICINA. Resolução $\mathbf{n}^{0} \mathbf{1 . 6 3 8}$ de julho de 2002. Define prontuário médico e torna obrigatória a criação da Comissão de Revisão de Prontuários nas instituições de saúde. Brasília: Diário Oficial União, 9 ago. 2002. p. 184-185. Disponível em: $<\underline{\text { https://sistemas.cfm.org.br/normas/visualizar/resolucoes/BR/2002/1638>. }}$.

LAHM, J. V; CARVALHO, D. R. Prontuário eletrônico do paciente: avaliação de usabilidade pela equipe de enfermagem. Cogitare Enfermagem, v. 20, n. 1, 2015. Disponível em: $<$ https://revistas.ufpr.br/cogitare/article/view/36485/24835>.

LIMA, V. S; et al. Prontuário eletrônico do cidadão: desafios e superações no processo de informatização. RE. SAÚD. DIGI. TEC. EDU., Fortaleza, CE, v. 3, número especial, p. 100-113. 2018.

Disponível

em:

$<\underline{\text { https://www.periodicos.ufc.br/resdite/article/view/39756/95752>. }}$.

MENDES, E. V. A modelagem das redes de atenção à Saúde. Secretaria de Estado de Saúde de Minas Gerais: Julho, 2007. Disponível em: $<$ https://www.nescon.medicina.ufmg.br/biblioteca/imagem/0260.pdf $>$.

MOURÃO, A. D. NEVES, J. T. R. Impactos da Implantação do Prontuário Eletrônico do Paciente sobre o Trabalho dos Profissionais de Saúde da Prefeitura Municipal de Belo Horizonte. Anais do Simpósio de Excelência em Gestão e Tecnologia; 2007 Out 22-24; Resende. Rio de Janeiro. Disponível em:

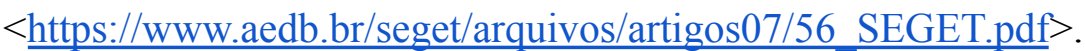

OLIVEIRA, J. F. Gestão de Tecnologias da Informação e da comunicação na Saúde: uma análise sobre o uso do prontuário eletrônico. Interface, v. 9, n. 1, 2013. Disponível em: $<$ http://www.spell.org.br/documentos/ver/13366/gestao-de-tecnologias-da-informacao-e-da-co muni--->.

PINTO, V. B. Prontuário eletrônico do paciente: documento técnico de Informação e comunicação do domínio da saúde, pesquisa sobre o Prontuário Eletrônico da Paciente. 


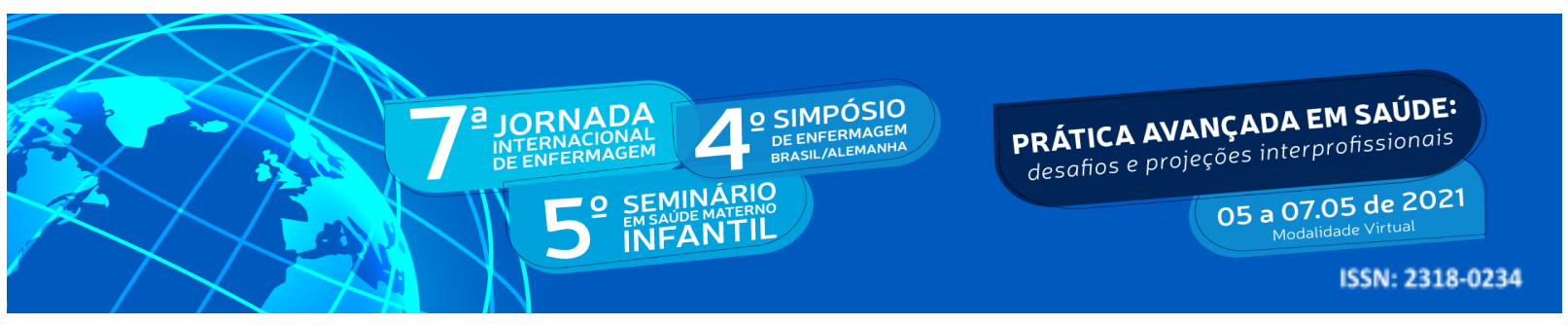

Revista eletrônica de biblioteconomia, n. 21, p. 34-48, 2006. Disponível em: $<\underline{\text { https://periodicos.ufsc.br/index.php/eb/article/view/1518-2924.2006v11n21p34/329> }}$

BRANDÃO, C.R. STRECK, D.R. A pesquisa participante e a partilha do saber - uma introdução. In: BRANDÃO, C.R. STRECK, D.R. (org) A pesquisa participante e a partilha do saber. Aparecida, SP: ideias e letras, 2006.

HOLLIDAY, O.J. Sistematização de experiências: algumas apreciações. In: BRANDÃO, C.R. STRECK, D.R. (org) A pesquisa participante e a partilha do saber. Aparecida, SP: ideias e letras, 2006. 